\title{
Hot Flashes and Carotid Intima Media Thickness among Midlife Women
}

Rebecca C. Thurston, PhDa,b, Kim Sutton-Tyrrell, DrPH ${ }^{b}$, Susan A. Everson-Rose, PhD, MPH $^{\mathrm{c}}$, Rachel Hess, MD, MSc ${ }^{\mathrm{b}, \mathrm{d}}$, Lynda H. Powell, MEd, PhD $^{\mathrm{e}}$, and Karen A. Matthews, $\mathrm{PhD}{ }^{\mathrm{a}, \mathrm{b}}$

aDepartment of Psychiatry, University of Pittsburgh School of Medicine

bDepartment of Epidemiology, University of Pittsburgh Graduate School of Public Health

'Department of Medicine, University of Minnesota School of Medicine

${ }^{d}$ Department of Medicine, University of Pittsburgh School of Medicine

eDepartment of Preventive Medicine, Rush University Medical Center

\begin{abstract}
Objective-Emerging evidence suggests associations between menopausal hot flashes and cardiovascular risk. Whether hot flashes are associated with intima media thickness (IMT) or IMT changes over time is unknown. We hypothesized that reported hot flashes would be associated with greater IMT cross-sectionally and with greater IMT progression over two years.
\end{abstract}

Methods-Participants were 432 women ages 45-58 at baseline participating in SWAN Heart, an ancillary study to the Study of Women's Health Across the Nation. Measures at the SWAN Heart baseline and follow-up visit two years later included a carotid artery ultrasound, reported hot flashes (past two weeks: none, 1-5, $\geq 6$ days), and a blood sample for measurement of estradiol.

Results-Women reporting hot flashes $\geq 6$ days in the prior two weeks had significantly higher IMT than women without hot flashes at baseline (mean difference(SE), $\mathrm{mm}=0.02(0.01), \mathrm{p}=0.03$ ) and follow-up (mean difference(SE), $\mathrm{mm}=0.02(0.01), \mathrm{p}=0.04)$ visits, controlling for demographic factors and cardiovascular risk factors. Reporting hot flashes at both study visits was associated with higher follow-up IMT relative to reporting hot flashes at neither visit (mean difference(SE), $\mathrm{mm}=0.03(0.01), \mathrm{p}=0.03)$. Associations between hot flashes and IMT largely remained after adjusting for estradiol. An interaction between hot flashes and obesity status was observed $(\mathrm{p}=0.05)$ such that relations between hot flashes and IMT were observed principally among overweight/obese women. Hot flashes were not associated with IMT progression.

Conclusions-These findings provided some indication that women reporting hot flashes $\geq 6$ days in the prior two weeks may have higher IMT than women without hot flashes, particularly for women who are overweight or obese. Further work should determine whether hot flashes mark adverse underlying vascular changes.

\section{Keywords}

atherosclerosis; women; hot flashes; vasomotor symptoms; epidemiology; cardiovascular disease

Corresponding author: Rebecca C. Thurston, PhD, 3811 O'Hara Street, Pittsburgh, PA 15213 (412) 648-9087, (412) $648-7160$ (fax), thurstonrc@upmc.edu.

All authors report no conflict of interest. 


\section{Introduction}

Over $70 \%$ of women report hot flashes during the menopausal transition.1 Hot flashes are associated with impairments in quality of life, 2 particularly sleep disruption 3 depressed mood, 4 and possibly memory problems.5 Most women with menopausal symptoms, principally hot flashes, seek treatment for them. 6

In addition to their quality of life implications, emerging research suggests associations between hot flashes and cardiovascular risk. In the Women's Health Initiative, the greatest cardiovascular risk associated with hormone therapy (HT) use was among the older postmenopausal women reporting moderate-severe hot flashes at baseline.7 A similar pattern of findings was observed in the Heart and Estrogen/Progestin Replacement Study (HERS), a study of older postmenopausal women with heart disease. 8 We found among midlife women participating in the Study of Women's Health Across the Nation (SWAN) Heart Study that hot flashes were associated with poorer flow mediated dilation and higher aortic calcification, 9 two subclinical cardiovascular disease (CVD) measures. These associations persisted controlling for CVD risk factors and estradiol (E2) concentrations.

However, contradictory findings exist. In one study, pulse wave velocity was inversely related to subjective hot flash severity ratings, 10 but markers of atherosclerosis were not included. In another study, endothelial function, but not carotid intima media thickness (IMT), differed between women with none to mild hot flashes relative to women with moderate to severe hot flashes.11 Given these suggestive yet not entirely consistent findings, there have been calls for increased research on links between hot flashes and CVD risk.12

In our present work, we examined relations between hot flashes and carotid IMT. IMT as measured by B-mode ultrasound is one of the most widely-used and well-validated measures of subclinical CVD.13 We hypothesized that hot flashes will be cross-sectionally associated with higher IMT, controlling for CVD risk factors and E2 concentrations, two key mechanisms that may link hot flashes to IMT.1, $14^{-} 16$ We also hypothesized that hot flashes will be associated with greater progression in IMT over two time points. Finally, we examined interactions between hot flashes and HT use, age, menopausal stage, and body mass index (BMI) in relation to IMT, as these factors can play key roles in relations between hot flashes and CVD risk. $7^{-9}$

\section{Methods}

\section{Study population}

SWAN is a cohort study designed to characterize the menopausal transition conducted at seven sites across the United States. Details of study design and procedures have been reported previously.17 At enrollment (1996-1997), SWAN participants $(n=3302)$ were aged 42-52 years, had an intact uterus and $\geq$ one ovary, were not pregnant or breast feeding, had menstruated within three months, and were not using oral contraceptives or HT. The study was approved by the Institutional Review Board at each site. Each participant provided written informed consent.

A subcohort of participants at Pittsburgh and Chicago SWAN sites participated in SWAN Heart, an ancillary study to assess cardiovascular risk. SWAN Heart included two assessments, the baseline exam occurring once during SWAN study years 4-7 (2001-2005) and the follow-up exam occurring two years later during SWAN study years 6-9 (2003-2008). Each SWAN Heart visit occurred within three months of the corresponding annual core SWAN assessment. By design,17 Pittsburgh and Chicago sites recruited only non-Hispanic White and Black women. Therefore, SWAN Heart participants described 
themselves as White or Black. Eligible SWAN participants were invited to participate in SWAN Heart; of these women, $76 \%$ enrolled. SWAN Heart exclusion criteria were pregnancy, hysterectomy or bilateral oophorectomy, reported CVD (prior myocardial infarction, angina, stroke, transient ischemia, peripheral vascular disease, other vascular disease including fibromuscular dysplasia, collagen vascular disease), diabetes, or medications to treat these conditions. Women who used HT (i.e., estrogen with or without progesterone and hormonal contraceptives) were also excluded, with one caveat. Women at the Pittsburgh site had been recruited for a subclinical CVD study at core SWAN visits 0 and 1 with similar study criteria. When SWAN Heart began during visits 4-7, the decision was made to enroll them in SWAN Heart, even if they did not meet certain study criteria. Thus, by the time these women underwent subclinical CVD assessments in SWAN Heart, 53 women were using HT. HT use was considered as covariate in all analyses.

Of the 588 SWAN Heart participants, 493 and 433 women provided information on hot flashes and underwent carotid ultrasound at baseline and follow-up, respectively. Sixty-one and 67 women were excluded from baseline and follow-up models, respectively, due to missing values on covariates or oophorectomy by the follow-up. Women excluded were more likely to be Black and to be smokers, and to have a higher BMI, systolic (SBP) and diastolic blood pressure (DBP), glucose, follow-up IMT, and lower physical activity (all p's $<0.05$ ) than women included. Missing BMI, BP, lipids, glucose, and smoking values were carried forward from the last completed assessment. Thus, samples to evaluate primary hypotheses were 432 women at baseline, 366 at follow-up, and 346 in models over the two visits.

\section{Design and Procedures}

Participants completed a standard protocol at SWAN entry and annually thereafter, including questionnaires, blood specimens, anthropometric measures, and blood pressure readings. Carotid ultrasonography was initiated during the SWAN Heart baseline visit and repeated at the SWAN Heart follow-up visit two years later.

\section{Intima Media Thickness}

IMT was assessed by B-mode ultrasonography of the left and right carotid arteries with a Hewlett-Packard 5500 scanner (Hewlett- Packard, Andover, Mass.) at the Chicago site and a Toshiba SSA- 270A scanner (Toshiba American Medical Systems, Tustin, Calif.) at the Pittsburgh site. Image quality was comparable between the two systems. Images were obtained from four locations of the left and right carotid arteries: the near and far walls of the distal common carotid artery, far wall of the bulb, and first $\mathrm{cm}$ of the far wall of the internal carotid artery. IMT measures were performed by electronically tracing the lumenintima interface and the media-adventitia interface across $1 \mathrm{~cm}$ segments in these locations. One measure is generated for each pixel, for a total of $~ 140$ measures per segment. The mean of average readings across the 8 locations comprised the average IMT. Readings were conducted at the University of Pittsburgh Ultrasound Research Laboratory under the direction of Dr Sutton-Tyrrell. Readers were recertified annually against the same set of scans to guard against reader drift. Replicate IMT readings were performed on 20 scans (intraclass correlation $=0.98$ ). Previous reports using the same laboratory and duplex scanning protocol have also shown high reproducibility. 18

\section{Hot flashes}

At the annual SWAN interviews corresponding to the SWAN Heart baseline and follow-up visits, participants reported the number of days in which hot flashes were experienced (not at all, 1-5 days, 6-8 days, 9-13 days, every day) in the two weeks prior to the interview. Women were categorized as experiencing hot flashes on none, $1-5$, or $\geq 6$ days in the past 
two weeks. To examine the stability of hot flashes across visits, women were also categorized as experiencing hot flashes at neither, one, or both visits. Night sweats were examined and showed similar yet attenuated patterns of associations in relation to IMT, but findings were largely nonsignificant and thereby are not reported here.

\section{Estradiol}

Measures were obtained from a morning fasting blood sample during the annual SWAN visit corresponding to SWAN Heart baseline. Subjects were scheduled for venipuncture on days 2-5 of a spontaneous menstrual cycle. Two attempts were made for a timed sample, obtained on $35 \%$ of participants. If a timed sample could not be obtained, a random fasting sample was taken. Blood was refrigerated prior to centrifugation 1-2 hours after phlebotomy, and serum aliquotted, frozen, and batched for shipment to the central laboratory. Samples were catalogued upon arrival and batches assayed monthly. Assays were performed on the ACS-180 automated analyzer (Bayer Diagnostics, Tarrytown, NY) utilizing a double-antibody chemiluminescent immunoassay with a solid phase anti-IgG immunoglobulin conjugated to paramagnetic particles, anti-ligand antibody, and competitive ligand labeled with dimethylacridinium ester. The assay modifies the rabbit anti-E2-6 ACS-180 immunoassay to increase sensitivity (lower limit of detection: $1.0 \mathrm{pg} / \mathrm{mL}$ ). Duplicate assays were conducted with results reported as the arithmetic mean for each subject, with a coefficient of variation of 3-12\%.

\section{Covariates}

Race/ethnicity, education ( $\leq$ high school, some college/vocational, $\geq$ college degree), and lipids were derived from the baseline SWAN interview and SWAN Heart exam. All other covariates were derived from the annual SWAN interview/exam corresponding to the SWAN Heart visit. Age was considered as a continuous variable. Race/ethnicity was determined in response to "How would you describe your primary racial or ethnic group?" Menopausal status, obtained annually from reported bleeding patterns, was categorized as premenopausal (bleeding in previous three months with no past year change in cycle predictability), early perimenopausal (bleeding the previous three months with decrease in cycle predictability in the past year), late perimenopausal ( $>3-<12$ months amenorrhea), or postmenopausal ( $\geq 12$ months amenorrhea). Due to small cell sizes, premenopausal and early perimenopausal women were combined for analysis. Women last classified as pre- or perimenopausal who reported taking hormones (oral contraceptives, oral estrogens and/or progestins, estrogen injections or patch) in the past year were considered indeterminate status due to the impact of hormone use, even if discontinued, on bleeding patterns. Women taking hormones within the past month, regardless of the last known menopausal status, were classified as HT users. Physical activity was assessed via a modified Kaiser Permanente Health Plan Activity Survey.19 CVD conditions (reported hypertension, and relevant to the follow up visit, angina, stroke, and heart attack) and CVD medications (reported use of medication for a heart condition or for blood pressure lowering in the past year) were combined into a single variable. Diabetes history/medication use, relevant to the follow up visit, was the report of having diabetes/current use of medications for treatment of diabetes. Anthropometric measures (height, weight, SBP, DBP) as well as total serum cholesterol, HDL, LDL, triglycerides, and glucose from a fasting blood sample were obtained using standard methods. BMI was calculated as weight $(\mathrm{kg}) / \mathrm{height}(\mathrm{m})^{2}$ and considered as a continuous covariate.

\section{Statistical analysis}

Variables were examined for distributions, outliers, and small cell sizes. HDL, triglycerides, glucose (baseline only), and E2 were log transformed prior to analysis due to evidence of skew. Univariate associations between hot flashes and covariates were evaluated via 
ANOVA, linear regression, and logistic regression. Multiple linear regression was used to evaluate cross-sectional associations between hot flashes and IMT at baseline and follow-up, as well as relations between hot flashes across visits and follow-up IMT or IMT progression. IMT progression was calculated as follow-up IMT-baseline IMT. IMT progression was alternatively evaluated by examining associations between hot flashes and follow-up IMT controlling for baseline IMT. Since there were no significant associations between hot flashes and IMT progression with either approach, nor when stratified by obesity status, data for progression models are not presented. Covariates were site, race, education, menopausal status, BMI, smoking status, diabetes status/medication use, CVD status/medication use, HT, SBP, DBP, HDL, LDL, triglycerides, glucose, and physical activity. For hormonal models, E2 was added to risk factor-adjusted models, with timing (cycle day) of blood draw also covaried. Due to collinearity between blood draw timing and menopausal status, only blood draw timing was included in statistical models. Interactions by age, race, HT, BMI, and menopausal stage were evaluated in primary models via cross-product interaction terms between hot flashes and each potential effect modifier. Analyses were performed with SAS (v.9.2, SAS Institute, Cary, NC). All tests were 2-sided, alpha=0.05.

\section{Results}

At the SWAN Heart baseline, women with hot flashes were older, less often White, had a lower education, were later in the menopausal transition, and had higher BMI, higher SBP, higher glucose, higher LDL, and lower E2 than women without hot flashes (Table 1). Fortysix percent of the women reported hot flashes at baseline and 54\% of women reported hot flashes at the follow-up. When considering the trajectory of hot flashes across visits, $37.9 \%$ $(n=131)$ of women had no hot flashes, 28.9\% $(n=100)$ had hot flashes at one visit, and $33.2 \%(\mathrm{n}=115)$ had hot flashes at both visits. Average (standard deviation (SD)) IMT was $0.67(0.086) \mathrm{mm}$ at the SWAN Heart baseline, and $0.71(0.093) \mathrm{mm}$ at the follow-up. Mean (SD) IMT progression across visits was $0.037(0.064) \mathrm{mm}$.

At baseline, having hot flashes $\geq 6$ days in the past two weeks was associated with higher IMT in risk factor-adjusted models; further adjustment for E2 somewhat attenuated these associations (Table 2). At the follow up visit, reporting hot flashes $\geq 6$ days in the past two weeks was associated with higher IMT in risk factor-adjusted models; these associations remained significant with adjustment for E2 (Table 3). Further, hot flashes at both visits were associated with higher follow-up IMT in both risk factor and E2-adjusted models. In contrast to risk-factor adjusted models, in minimally-adjusted (age, site, race) models, associations between hot flashes and IMT showed a similar, but less pronounced pattern of associations at baseline (hot flashes $\geq 6$ days versus none: $b(\mathrm{SE})=0.01(0.01), \mathrm{p}=0.18,1-5$ days versus none: $b(S E)=0.01$ (0.009), $p=0.27$ ), follow-up (hot flashes $\geq 6$ days versus none: $\mathrm{b}(\mathrm{SE})=0.02(0.01), \mathrm{p}=0.11,1-5$ days versus none: $\mathrm{b}(\mathrm{SE})=0.02(0.01), \mathrm{p}=0.04)$ and across visits (hot flashes across both visits versus no visits: $b(S E)=0.02(0.01), p=0.06$; one visit versus no visits: $b(S E)=0.02(0.01) p=0.10)$. Thus, rather than accounting for observed associations, adjusting for covariates made associations more pronounced. There were no significant associations between hot flashes and IMT progression (e.g., in risk factoradjusted models, baseline hot flashes $\geq 6$ days versus none: $b$ (SE) $=0.009(0.01), p=0.37$; hot flashes across both visits versus no visits: $b(\mathrm{SE})=-0.001(0.009), \mathrm{p}=0.91)$. See also Supplementary Table 1 in the Appendix.

No interactions between age, race, HT use, or menopausal status and hot flashes in relation to IMT were observed. There was indication of an interaction between hot flashes and BMI in relation to baseline $(\mathrm{p}=0.05)$ and follow-up IMT $(\mathrm{p}=0.05)$ and to a lesser extent for hot flashes across visits and follow-up IMT $(\mathrm{p}=0.10)$. Relations between hot flashes and IMT were most pronounced among overweight/obese women (Figure 1). 
Findings for night sweats showed a similar, yet attenuated pattern to those for hot flashes in risk factor-adjusted models at baseline (night sweats none versus $\geq 6$ nights in past two weeks $\mathrm{b}(\mathrm{SE})=0.02(0.01), \mathrm{p}=0.03$ ), follow-up (night sweats none versus $\geq 6$ nights in past two weeks: $b(\mathrm{SE})=0.02(0.01), \mathrm{p}=0.11$ ), and across visits (night sweats at both versus neither visit: $b(\mathrm{SE})=0.02(0.01), \mathrm{p}=0.19)$. See also Supplementary Tables 2, 3 in the Appendix.

\section{Discussion}

This study suggests that women reporting hot flashes $\geq 6$ days in the prior two weeks may have higher IMT than women reporting no hot flashes. While findings were not entirely consistent, greater hot flash reporting was generally associated with higher IMT, with associations apparent after controlling for traditional CVD risk factors. E2 accounted for a portion, but not all, of the associations between hot flashes and IMT. Although the magnitude of the association between hot flashes and IMT was modest, it was consistent with effect sizes observed for obesity, smoking, and menopausal status in relation to IMT in this sample. Moreover, associations were principally observed among overweight and obese women. Hot flashes were not associated with IMT progression.

IMT is one of the most widely-used and well-validated measures of subclinical CVD. Higher IMT has been associated with elevated CVD risk factors, atherosclerosis, prevalent CVD, and prospectively with incident CVD events.13, 20 The predictive value of IMT is particularly evident among low risk individuals, 21 such as the women in this study. Thus, when these results are considered in conjunction with our prior work with this cohort, 9 we see that SWAN Heart participants reporting hot flashes, particularly every other day or more, had elevated subclinical CVD, including higher IMT, aortic calcification, and poorer flow mediated dilation than women without hot flashes.

Our findings are largely consistent with prior work from clinical trials $7^{-} 8$ and observational epidemiologic studies9, 22 showing associations between hot flashes and cardiovascular risk. However, there are inconsistencies in this literature. In one study, pulse wave velocity was inversely related to subjective hot flash severity ratings, 10 although in that study there were few women without hot flashes and markers of atherosclerosis were not included. In another study, having both hot flashes and night sweats was associated with reduced mortality risk, but relations were null when adjusting for confounders.23 Finally, another study found that women with moderate to severe hot flashes had poorer endothelial function, but not IMT, than women with none/mild hot flashes.11 Notably, their ability to detect associations for IMT, versus endothelial dysfunction typically evident earlier in the disease process, was limited by their exceptionally healthy sample and very low IMT levels.

Associations between hot flashes and IMT in this study were observed primarily among overweight and obese women. Notably, the overweight/obese women in this sample had higher mean IMT and variability in IMT than lean women, increasing the likelihood of detecting associations. Further, overweight and obese women also have more hot flashes relative to lean women.1 Finally, this work may suggest a synergistic effect between hot flashes and existing CVD risk factors with respect to IMT, which should be considered in future work. Consistent with our prior research among midlife women, 9 no interactions were observed between hot flashes and age, menopausal stage, or HT use, indicating that findings extend across these groups.

The mechanisms underlying links between hot flashes and cardiovascular risk require greater elucidation. Current models characterize hot flashes as thermoregulatory events occurring in the context of menopausal endocrine changes.24 However, the physiology of hot flashes is not well-understood and many other systems have been implicated. The low 
E2 that may play a role in the etiology of hot flashes is generally associated with a poorer cardiovascular profile.15`16 However, adjusting for endogenous E2 attenuated, but did not fully eliminate the associations between hot flashes and IMT. Further, CVD risk factors such as smoking and obesity are risk factors for hot flashes, 1,14 and some studies have shown adverse metabolic1 or BP profiles14 with hot flashes. Residual confounding is always possible in observation research, although it is notable that relations between hot flashes and IMT were not reduced but in fact strengthened controlling for CVD risk factors. The reason for this pattern of associations is not entirely clear, but does not support the notion that the CVD risk factors accounted for these relations. Finally, our prior research showed acute decreases in cardiac vagal control during hot flashes;25 and ours9 and others' 11 prior work suggested poorer endothelial function associated with hot flashes. These systems may be relevant to understanding the associations observed here. Given evidence of links between hot flashes and cardiovascular risk, further investigation of potential underlying mechanisms is needed.

A unique aspect of this study is its consideration of relations between hot flashes and IMT in the longitudinal setting. We observed cross-sectional relations between hot flashes and IMT, as well as reporting hot flashes at both visits being associated with higher IMT. However, hot flashes were not associated with IMT progression. IMT was assessed two years apart, and while statistically significant progression was observed, the magnitude of this change was small. Calculating IMT progression includes error from both baseline and follow-up measurements, limiting the ability to detect differences in progression by hot flash status. Further, the reduction in E2 that accompanies menopause can be associated with vascular adaptation 16 that would impact IMT and make detection of clear changes between these two time points challenging.26 Thus, these results support a link between hot flashes and subclinical CVD but not a role for hot flashes in the progression of IMT, although further research on progression over longer follow up times and into older ages is warranted.

Several limitations deserve mention. Hot flashes were characterized using a brief, self-report measure. While common in epidemiologic investigations, these measures provide limited information about hot flashes and are subject to memory biases. Future work should utilize more detailed hot flashes measures. Moreover, E2 was characterized via a single annual blood sample, which may not have fully characterized E2 exposure, particularly among perimenopausal women experiencing wide hormonal fluctuations. While daily assessments were not feasible in this epidemiologic study, more detailed assessments should be undertaken in future work. Further, women excluded had a higher risk profile with respect to cardiovascular disease and possibly hot flashes than women included; therefore associations may have been underestimated here. Finally, power may have been insufficient to fully assess interactions by relevant subject characteristics; these sub-analyses should be undertaken in future work with larger sample sizes and across a range of menopausal stages not fully represented here (i.e., premenopausal women, late postmenopausal women). This work points to the need for future investigations designed to more fully characterize relations between hot flashes and cardiovascular risk.

The present investigation has several strengths. It is the first to examine relations between hot flashes and IMT in a community-dwelling sample of midlife women, demonstrating significant associations with this widely-validated subclinical CVD measure. The study included sizeable numbers of Black and White women, indicating that associations extend to both groups. It allowed consideration of associations longitudinally, showing the pattern and consistency of associations between hot flashes and IMT over time. Finally, the study included a sample of well-characterized women that has been followed throughout the menopausal transition. 


\section{Conclusions}

The present work adds to the growing body of literature concerning hot flashes and CVD. Notably, hot flashes occur at a time of life characterized by increasing CVD risk for women. The precise nature of relations between hot flashes and cardiovascular risk requires greater explication. With additional research, investigators will be able to more conclusively determine whether hot flashes mark adverse underlying vascular changes, potentially signaling to clinicians the need for more aggressive traditional CVD risk factor reduction among women with hot flashes. These findings also may have implications for understanding the physiology of hot flashes, suggesting consideration of vascular involvement in hot flashes. This work supports the importance of continued work to further understand the role of hot flashes in cardiovascular risk.

\section{Supplementary Material}

Refer to Web version on PubMed Central for supplementary material.

\section{Acknowledgments}

The Study of Women's Health Across the Nation (SWAN) has grant support from the National Institutes of Health (NIH), DHHS, through the National Institute on Aging (NIA), the National Institute of Nursing Research (NINR) and the NIH Office of Research on Women's Health (ORWH) (Grants NR004061; AG012505, AG012535, AG012531, AG012539, AG012546, AG012553, AG012554, AG012495). SWAN Heart was supported by grants from the NIH through the National Heart Lung and Blood Institute (HL065581, HL065591). The content of this article is solely the responsibility of the authors and does not necessarily represent the official views of the NIA, NINR, ORWH or the NIH.

Clinical Centers: Rush University, Rush University Medical Center, Chicago, IL - Howard Kravitz, PI 2009 present, Lynda Powell, PI 1994 - 2009; University of Pittsburgh, Pittsburgh, PA - Karen Matthews, PI.

NIH Program Office: National Institute on Aging, Bethesda, MD - Sherry Sherman 1994 - present; Marcia Ory 1994 - 2001; National Institute of Nursing Research, Bethesda, MD - Project Officer.

Central Laboratory: University of Michigan, Ann Arbor-Daniel McConnell (Central Ligand Assay Satellite Services).

Coordinating Center: New England Research Institutes, Watertown, MA-Sonja McKinlay, PI 1995-2001; University of Pittsburgh, Pittsburgh, PA-Kim Sutton-Tyrrell, PI 2001-present.

Steering Committee: Susan Johnson, Current Chair; Chris Gallagher, Former Chair. Dr. Thurston received grant support from the NIH through the National Institute on Aging (AG029216).

This work is supported by NIH Grants NR004061; AG012505, AG012535, AG012531, AG012539, AG012546, AG012553, AG012554, AG012495, HL065581, HL065591, and AG029216.

\section{References}

1. Gold E, Colvin A, Avis N, et al. Longitudinal analysis of vasomotor symptoms and race/ethnicity across the menopausal transition: Study of Women's Health Across the Nation (SWAN). Am J Public Health. 2006; 96(7):1226-35. [PubMed: 16735636]

2. Avis NE, Ory M, Matthews KA, Schocken M, Bromberger J, Colvin A. Health-related quality of life in a multiethnic sample of middle-aged women: Study of Women's Health Across the Nation (SWAN). Med Care. 2003; 41(11):1262-76. [PubMed: 14583689]

3. Kravitz HM, Ganz PA, Bromberger J, Powell LH, Sutton-Tyrrell K, Meyer PM. Sleep difficulty in women at midlife: a community survey of sleep and the menopausal transition. Menopause. 2003; 10(1):19-28. [PubMed: 12544673] 
4. Bromberger JT, Matthews KA, Schott LL, et al. Depressive symptoms during the menopausal transition: the Study of Women's Health Across the Nation (SWAN). J Affect Disord. 2007; 103(1-3):267-72. [PubMed: 17331589]

5. Maki PM, Drogos LL, Rubin LH, Banuvar S, Shulman LP, Geller SE. Objective hot flashes are negatively related to verbal memory performance in midlife women. Menopause. 2008; 15(5):84856. [PubMed: 18562950]

6. Williams RE, Kalilani L, DiBenedetti DB, Zhou X, Fehnel SE, Clark RV. Healthcare seeking and treatment for menopausal symptoms in the United States. Maturitas. 2007; 58(4):348-58. [PubMed: 17964093]

7. Rossouw JE, Prentice RL, Manson JE, et al. Postmenopausal hormone therapy and risk of cardiovascular disease by age and years since menopause. JAMA. 2007; 297(13):1465-77. [PubMed: 17405972]

8. Huang AJ, Sawaya GF, Vittinghoff E, Lin F, Grady D. Hot flushes, coronary heart disease, and hormone therapy in postmenopausal women. Menopause. 2009

9. Thurston RC, Sutton-Tyrrell K, Everson-Rose SA, Hess R, Matthews KA. Hot flashes and subclinical cardiovascular disease: Findings from the Study of Women's Health Across the Nation Heart Study. Circulation. 2008; 118(12):1234-40. [PubMed: 18765392]

10. Tuomikoski P, Ebert P, Groop PH, et al. Evidence for a role of hot flushes in vascular function in recently postmenopausal women. Obstet Gynecol. 2009; 113(4):902-8. [PubMed: 19305337]

11. Bechlioulis A, Kalantaridou SN, Naka KK, et al. Endothelial Function, But Not Carotid IntimaMedia Thickness, Is Affected Early in Menopause and Is Associated with Severity of Hot Flushes. J Clin Endocrinol Metab. 2010

12. Allison MA, Manson JE. The complex interplay of vasomotor symptoms, hormone therapy, and cardiovascular risk. Menopause. 2009; 16(4):619-20. [PubMed: 19503007]

13. Lorenz MW, Markus HS, Bots ML, Rosvall M, Sitzer M. Prediction of clinical cardiovascular events with carotid intima-media thickness: a systematic review and meta-analysis. Circulation. 2007; 115(4):459-67. [PubMed: 17242284]

14. Gast GC, Grobbee DE, Pop VJ, et al. Menopausal complaints are associated with cardiovascular risk factors. Hypertension. 2008; 51(6):1492-8. [PubMed: 18391100]

15. Sader MA, Celermajer DS. Endothelial function, vascular reactivity and gender differences in the cardiovascular system. Cardiovasc Res. 2002; 53(3):597-604. [PubMed: 11861030]

16. Wildman RP, Colvin AB, Powell LH, et al. Associations of endogenous sex hormones with the vasculature in menopausal women: the Study of Women's Health Across the Nation (SWAN). Menopause. 2008; 15(3):414-21. [PubMed: 18209686]

17. Sowers, M.; Crawford, S.; Sternfeld, B., et al. SWAN: A multicenter, multiethnic, communitybased cohort study of women and the menopausal transition.. In: Lobo, RA.; Kelsey, J.; Marcus, R.; Lobo, AR., editors. Menopause: Biology and Pathology. Academic Press; New York: 2000. p. 175-88.

18. Sutton-Tyrrell K, Wolfson SK, Thompson T, Kelsey SF. Measurement variability in duplex scan assessment of carotid atherosclerosis. Stroke. 1992; 23:215-20. [PubMed: 1561650]

19. Ainsworth BE, Sternfeld B, Richardson MT, Jackson K. Evaluation of the kaiser physical activity survey in women. Med Sci Sports Exerc. 2000; 32(7):1327-38. [PubMed: 10912901]

20. O'Leary DH, Polak JF, Kronmal RA, Manolio TA, Burke GL, Wolfson SK Jr. Carotid-artery intima and media thickness as a risk factor for myocardial infarction and stroke in older adults. Cardiovascular Health Study Collaborative Research Group. N Engl J Med. 1999; 340(1):14-22. [PubMed: 9878640]

21. Stein JH, Korcarz CE, Hurst RT, et al. Use of carotid ultrasound to identify subclinical vascular disease and evaluate cardiovascular disease risk: a consensus statement from the American Society of Echocardiography Carotid Intima-Media Thickness Task Force. Endorsed by the Society for Vascular Medicine. J Am Soc Echocardiogr. 2008; 21(2):93-111. quiz 89-90. [PubMed: 18261694]

22. Thurston R, Kuller L, Edmundowicz D, Matthews K. Hot flashes and aortic calcification among postmenopausal women. Menopause. 2010; 17(2):256-61. [PubMed: 20042895] 
23. Svartberg J, von Muhlen D, Kritz-Silverstein D, Barrett-Connor E. Vasomotor symptoms and mortality: the Rancho Bernardo Study. Menopause. 2009; 16(5):888-91. [PubMed: 19421076]

24. Freedman RR. Pathophysiology and treatment of menopausal hot flashes. Semin Reprod Med. 2005; 23(2):117-25. [PubMed: 15852197]

25. Thurston R, Christie I, Matthews K. Hot flashes and cardiac vagal control: A link to cardiovascular risk? Menopause. 2010; 17(3):456-61. [PubMed: 20042892]

26. Eigenbrodt ML, Bursac Z, Rose KM, et al. Common carotid arterial interadventitial distance (diameter) as an indicator of the damaging effects of age and atherosclerosis, a cross-sectional study of the Atherosclerosis Risk in Community Cohort Limited Access Data (ARICLAD), 1987-89. Cardiovasc Ultrasound. 2006; 4:1. [PubMed: 16390545] 


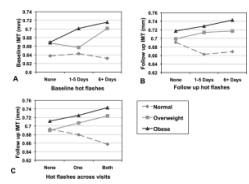

Figure 1. Relation between hot flashes at baseline, follow-up, and across visits in relation to IMT by obesity status

A: Relation between hot flashes and IMT by obesity status at baseline; B: Relation between hot flashes and IMT by obesity status at follow up; C: Relation between hot flashes across visits and follow-up IMT by obesity status. Models adjusted for age, site, race, education, menopausal status, BMI, smoking status, diabetes status/medication use (follow-up), CVD status/medication use, HT use, SBP, DBP, HDL, LDL, triglycerides, glucose, physical activity. 
Table 1

Baseline sample characteristics by baseline hot flash status, SWAN Heart Study

\begin{tabular}{|c|c|c|c|}
\hline & \multicolumn{3}{|c|}{ Baseline hot flashes status } \\
\hline & None $(\mathrm{N}=239)$ & 1-5 days $(\mathrm{N}=126)$ & $6+$ days $(N=77)$ \\
\hline Age, $\mathrm{M}(\mathrm{SD})^{* \dagger}$ & $50.1(2.7)$ & $50.8(3.0)$ & $51.1(2.8)$ \\
\hline \multicolumn{4}{|l|}{$\operatorname{Race}(\mathrm{n}, \%)^{\dagger}$} \\
\hline Black & $71(29.7)$ & $56(44.4)$ & $29(37.7)$ \\
\hline White & $168(70.3)$ & $70(55.6)$ & $48(62.3)$ \\
\hline \multicolumn{4}{|l|}{ Education $(\mathrm{n}, \%)^{* \dagger}$} \\
\hline High school or less & $35(14.6)$ & $18(14.3)$ & $16(20.8)$ \\
\hline Some college/ associates & $61(25.5)$ & $49(38.9)$ & $29(37.7)$ \\
\hline College or higher & $143(59.8)$ & $59(46.8)$ & $32(41.6)$ \\
\hline \multicolumn{4}{|l|}{ Menopausal status $(\mathrm{n}, \%)^{* \dagger}$} \\
\hline Premenopausal ${ }^{\dagger}$ & $33(13.8)$ & $5(4.0)$ & $1(1.3)$ \\
\hline Early perimenopausal ${ }^{\ddagger}$ & $124(51.9)$ & $56(44.4)$ & $19(24.7)$ \\
\hline Late perimenopausal & $21(8.8)$ & $19(15.1)$ & $9(11.7)$ \\
\hline Postmenopausal & $47(19.7)$ & $42(33.3)$ & $42(54.6)$ \\
\hline Indeterminate & $14(5.9)$ & $4(3.2)$ & $6(7.8)$ \\
\hline $\mathrm{BMI}, \mathrm{M}(\mathrm{SD})^{\dagger}$ & $28.2(5.8)$ & $30.4(6.2)$ & $28.5(5.5)$ \\
\hline Smoking status (n, \% yes) & $32(13.4)$ & $20(15.9)$ & $15(19.5)$ \\
\hline Physical activity, M (SD) & $8.1(1.7)$ & $8.0(1.8)$ & $7.9(1.7)$ \\
\hline $\mathrm{SBP}, \mathrm{M}(\mathrm{SD})^{\dagger}$ & $117.5(16.4)$ & $122.3(18.1)$ & $118.1(14.3)$ \\
\hline $\mathrm{DBP}, \mathrm{M}(\mathrm{SD})$ & $75.2(9.8)$ & $77.1(10.7)$ & $75.4(8.8)$ \\
\hline $\mathrm{LDL}, \mathrm{M}(\mathrm{SD})^{*}$ & $117.0(33.5)$ & $120.8(28.9)$ & $126.0(37.5)$ \\
\hline HDL, M (SD) & $58.0(15.6)$ & $57.4(12.8)$ & $59.1(14.4)$ \\
\hline Triglycerides, M (SD) & $113.0(62.8)$ & $112.9(50.8)$ & $117.1(62.9)$ \\
\hline Glucose, $\mathrm{M}(\mathrm{SD})^{\dagger}$ & $89.2(19.3)$ & $91.8(11.0)$ & $92.2(16.4)$ \\
\hline Anti-hypertensive medication use (n, \% yes) & $44(18.4)$ & $20(15.9)$ & $12(15.6)$ \\
\hline HT use (n, \% yes) & $31(13.0)$ & $15(11.9)$ & $7(9.1)$ \\
\hline Estradiol, pg/mL, M (SD) ${ }^{* \dagger}$ & $79.6(97.9)$ & $48.8(63.6)$ & $31.7(41.3)$ \\
\hline
\end{tabular}

HDL, triglycerides, glucose, estradiol log transformed for analysis

6+days vs. none $\mathrm{p}<0.05$

${ }^{\dagger} 1-5$ days versus none $\mathrm{p}<0.05$

* premenopausal and early perimenopausal groups combined for analysis 
Table 2

Association between hot flashes and IMT at the SWAN Heart baseline visit

\begin{tabular}{|c|c|c|c|c|}
\hline & \multicolumn{4}{|c|}{ Baseline IMT } \\
\hline & \multicolumn{2}{|l|}{ Model 1} & \multicolumn{2}{|l|}{ Model 2} \\
\hline & Regression coefficient $^{\dagger}$ (SE) & $\mathbf{p}$ & Regression coefficient $^{\dagger}(\mathrm{SE})$ & $\mathbf{p}$ \\
\hline \multicolumn{5}{|c|}{ Hot flashes at baseline } \\
\hline None & -- & & -- & \\
\hline $1-5$ days & $0.01(0.01)$ & 0.33 & $0.01(0.01)$ & 0.36 \\
\hline $6+$ days & $0.02(0.01)$ & 0.03 & $0.02(0.01)$ & 0.13 \\
\hline
\end{tabular}

Model 1 covariates: age, site, race, education, menopausal status, BMI, smoking status, anti-hypertensive medication use, HT use, SBP, DBP, HDL, LDL, triglycerides, glucose, physical activity

Model 2 covariates: age, site, race, education, BMI, smoking status, anti-hypertensive medication use, HT use, SBP, DBP, HDL, LDL, triglycerides, glucose, physical activity, cycle day of blood draw, estradiol

${ }^{\dagger}$ Regression coefficient corresponds to mean IMT difference (mm) from referent of no hot flashes 
Table 3

Association between hot flashes at the SWAN Heart follow up visit and across baseline and follow up visits in relation to IMT at the follow up visit

\begin{tabular}{|c|c|c|c|c|}
\hline & \multicolumn{4}{|c|}{ IMT at follow up } \\
\hline & \multicolumn{2}{|l|}{ Model 1} & \multicolumn{2}{|l|}{ Model 2} \\
\hline & Regression coefficient $^{\dagger}$ (SE) & $\mathbf{p}$ & Regression coefficient $^{\dagger}$ (SE) & $\mathbf{p}$ \\
\hline \multicolumn{5}{|l|}{ Hot flashes at follow up } \\
\hline None & -- & & -- & \\
\hline $1-5$ days & $0.02(0.01)$ & 0.09 & $0.02(0.01)$ & 0.14 \\
\hline $6+$ days & $0.02(0.01)$ & 0.04 & $0.03(0.01)$ & 0.02 \\
\hline \multicolumn{5}{|l|}{ Hot flashes across visits } \\
\hline None & -- & & -- & \\
\hline Hot flashes at one visit & $0.02(0.01)$ & 0.20 & $0.02(0.01)$ & 0.13 \\
\hline Hot flashes at both visits & $0.03(0.01)$ & 0.03 & $0.03(0.01)$ & 0.03 \\
\hline
\end{tabular}

Model 1 covariates: age, site, race, education, menopausal status, BMI, smoking status, SBP, DBP, HDL, LDL, triglycerides, glucose, physical activity, diabetes status/medication use, CVD status/medication use, HT use

Model 2 covariates: age, site, race, education, BMI, smoking status, SBP, DBP, HDL, LDL, triglycerides, glucose, physical activity, diabetes status/medication use, CVD status/medication use, HT use, cycle day of blood draw, estradiol

Note: Hot flashes at follow up and hot flashes across visits modeled in separate models

${ }^{\dagger}$ Regression coefficient corresponds to mean IMT difference (mm) from referent of no hot flashes 